\title{
Links between surface magnetic fields, abundances, and surface rotation in clusters and in the field
}

\author{
Norbert Przybilla \\ Institute for Astro- and Particle Physics, University of Innsbruck, \\ Technikerstr. 25/8, A-6020 Innsbruck, Austria \\ email: norbert.przybilla@uibk.ac.at
}

\begin{abstract}
Theory predicts that hydrodynamical instabilities transport angular momentum and chemical elements in rotating massive stars. An interplay of rotation and a magnetic field affects these transport processes. The complexity of the problem imposes that a comprehensive description cannot be developed on theoretical grounds alone, progress in the understanding of the evolution of massive stars has to be guided by observations. The challenge lies both in the derivation of accurate and precise observational constraints as well as in the extraction of the relevant information for identifying possible correlations - like between surface magnetic fields, abundances, and surface rotation - from a multivariate function of the many parameters involved. I review the most important steps recently made based on detailed studies of massive stars both in the field and in clusters towards finding such links that ultimately may guide the further development of the models.
\end{abstract}

Keywords. stars: abundances, stars: early-type, stars: evolution, stars: magnetic fields, stars: rotation

\section{Introduction}

The first grids of evolution models for rotating massive stars (Meynet \& Maeder 2000; Heger \& Langer 2000) indicated changes in all the outputs when compared to classical models: lifetimes, evolution scenarios, nucleosynthesis, etc. They were very successful in explaining many observed phenomena, however many issues still lack a comprehensive understanding, like the ratio of blue-to-red supergiants in galaxies, supernova channels, long-soft $\gamma$-ray burst progenitors and the rotation rates of stellar remnants. Important in this context is that many uncertainties remain in the input physics of the models, e.g. in the mass-loss rates, in the nature of transport mechanisms and the effects of magnetic fields, which furthermore may be generated by some dynamo effect or be of fossil nature (e.g. Langer 2014). In particular challenging are quantitative predictions for the postmain sequence evolution, but one has to keep in mind that the basis for the further developments is already laid on the main-sequence by the details of the core evolution. The topic is of such complexity that progress requires strong guidance by observation.

The most sensitive variables for the comparison with observation are light element abundances, in particular nitrogen, which shows much larger variations like carbon, oxygen or helium (for recent results see e.g. Ekström et al. 2012; Chieffi \& Limongi 2013). Boron is also a highly sensitive indicator in particular for rotational mixing in the early evolution of massive stars (e.g. Frischknecht et al. 2010), however the determination of $\mathrm{B}$ abundances requires UV spectroscopy, therefore few results are available.

The development of the surface abundances, e.g. the nitrogen excess, is a multivariate function depending on mass $M$, age $\left(\tau_{\text {evol }}\right)$, projected rotational velocity $(v \sin i)$, 
metallicity $(Z)$ and multiplicity (Maeder et al. 2009): $\Delta(\mathrm{N} / \mathrm{H})=f\left(M, \tau_{\text {evol }}, v \sin i, Z\right.$, multiplicity). On the modeling side further 'variables' complicate the problem, in particular in form of the input physics considered and the values adopted for various input parameters like e.g. the overshoot parameter or diffusion coefficients. Therefore, theory needs to be guided by observation if meaningful models shall be developed. Observational constraints should not be only accurate and precise, but they should also be restricted in input parameter range as much as possible in order to facilitate the extraction of relevant information.

Massive stars in clusters should therefore be preferred as targets, as they have the same age and the same initial chemical composition, and they are located at the same distance. However, observationally they are more challenging for high-S/N spectroscopy because any Galactic cluster with a significant massive star population is much more distant than the numerous massive stars in the nearby field and associations, which consequently can be studied in greater detail. Ideally, a statistical approach is used to compare observations with models, employing population synthesis (e.g. Georgy et al. 2014; Schneider et al. 2014), and to find correlations between observational variables (Aerts et al. 2014).

The present work aims at reviewing and evaluating the current status in the literature on observational constraints for establishing a possible correlation between surface magnetic fields, abundances, and rotation. These are required for guiding the inclusion of the interaction of rotation with magnetic fields in models of massive star evolution (e.g. Heger et al. 2005; Maeder \& Meynet 2005; Meynet et al. 2011; Brott et al. 2011).

\section{Magnetic fields in massive stars}

The presence of magnetic fields in massive stars is difficult to be established. Interior magnetic fields may be ubiquitous in differentially rotating stars where the Tayler-Spruit dynamo (Spruit 2002) may be operational. However, they do not reach the stellar surface to become observable (Maeder \& Meynet 2005). Their effects on the evolution of rotating massive stars (enhanced transport of angular momentum and chemical elements) can therefore be constrained only indirectly. Small-scale magnetic fields may also be generated in a sub-surface convection zone. They may reach the surface, manifesting as hot spots that give rise to discrete absorption components in the stellar wind (Cantiello et al. 2009). The sub-surface convection zone may be important for stellar evolution by inducing wind clumping, which impacts the mass-loss rates (see e.g. Puls, these proceedings).

Large-scale magnetic fields of fossil nature - either a relic of the interstellar magnetic field present during star formation, or generated in a binary merger - are the ones that can be detected by spectropolarimetry. Detections of a surface magnetic field require much higher S/N (>500) than typically achieved for classical quantitative spectroscopy. Therefore, limiting magnitudes for spectropolarimetric studies are significantly lower than for spectroscopy, and detections near the sensitivity limit are often not undisputed.

The knowledge about the occurrence of magnetism in hot, massive stars has benefited tremendously in the past years from large observational surveys like MiMeS (Wade et al. 2014) or the complementary BOB survey (Morel et al., these proceedings) in addition to individual PI projects (e.g. Hubrig et al. 2011). An extensive (though in the meantime no longer complete) list of magnetic OB stars is given by Petit et al. (2013). Overall, an incidence of surface magnetic fields in about $7 \%$ of the OB stars is indicated, with dipole field strengths varying from several hundred $\mathrm{G}$ to $\sim 20 \mathrm{kG}$ (Wade et al. 2014) and a likely extension towards lower field strengths at increased detection sensitivity (Morel et al., these proceedings). The full characterisation of the properties of magnetic fields is much more demanding than the detection, such that results are available only for a limited number of objects (e.g. Donati et al. 2006; Neiner et al. 2012; Henrichs et al. 2013). 


\section{Towards correlations between surface magnetic fields, abundances and rotational velocity in $O B$ stars: state-of-the-art}

Despite the importance of elemental abundances as the most sensitive observational constraints on evolution models, relatively little is known on light element abundances (He, B, CNO) in magnetic massive stars. The topic was addressed first by Morel et al. (2008), suggesting a higher incidence of chemical peculiarities in early B-type stars with (weak) magnetic fields, which in several cases could also be confirmed as genuine slow rotators by determining rotational periods either from the occurrence of phase-locked UV wind line-profile variations, or from asteroseismic modelling. N-rich objects were found to be B-poor, in agreement with expectations from theory. Note that this study is the only one considering boron abundances to date. Further studies found additional N-rich magnetic early-B and late-O stars (Morel 2012; Henrichs et al. 2012), but also four Nnormal magnetic stars (Morel 2011; Fossati et al. 2014). A strict correlation between the presence of magnetic fields and N-enrichment appears no longer assumable, additional parameters seem to play a rôle as well. The initial angular momentum is crucial, and possibly the star's feasibility to retain differential rotation in the presence of a large-scale magnetic field, vs. rigid rotation (Meynet et al. 2011). In particular, magnetic stars with fossil fields (relics of the interstellar-medium field) that were born with negligible angular momentum are not expected to show mixing signatures.

It is interesting in this context that no signatures of CNO-mixing were found in the components of detached eclipsing OB-type binaries so far (for a summary see Pavlovski \& Southworth 2013). Likewise, magnetic fields appear to be absent in massive binaries as indicated by the BinaMIcS project (Alecian et al., these proceedings).

Several O-type stars were analysed by Martins et al. (2012a) in order to investigate observational effects of magnetism in more massive objects. For the majority of the stars, slow rotation was found and N-enrichment beyond the values typical for mixed early Bstars. Note that HD 57682 (O9 IV) is in common with the study of Morel (2011), however a nitrogen abundance higher by $\sim 0.6$ dex was derived, suggesting it to be N-rich. This discrepancy needs to be resolved by further investigations. The sample of Martins et al. (2012a) includes three Of?p stars, the magnetic stars that have the strongest fields among O stars. They show high levels of surface N-enrichment, yet not unusually high compared to other O stars. They are also all slow rotators (Wade et al. 2012, and references therein), apparently a consequence of magnetic braking (Ud-Doula et al. 2009).

$\zeta$ Ori A (O9.5 I) is the only magnetic hot supergiant known to date. It is a N-normal fast rotator (Bouret et al. 2008; Martins et al. 2012a), which has been identified as a doublelined spectroscopic binary (SB2) by Hummel et al. (2013). The supergiant component $\zeta$ Ori Aa has been proven to be the magnetic star (Blazère et al., these proceedings).

As mentioned in the previous section, internal dynamo-generated magnetic fields may be ubiquitous among massive stars. It is therefore equally important to investigate possible correlations between rotation and surface abundances for samples of stars without detected surface fields. The by far most extensive study in the past years of $\sim 300$ cluster early B-stars was the FLAMES Survey of Massive Stars (Hunter et al. 2009). An unexpectedly large number of slowly-rotating N-rich stars was found, as well as a class of non-enriched rapid rotators. Thus, many objects appear to remain unexplained by evolution models for single stars, however see Sect. 5. Nitrogen surface abundances in Galactic O stars indicate a wider range of enrichment compared to B stars and a clear trend toward more enrichment in higher mass stars (Martins et al. 2012b). The bulk of the LMC O-stars studied by Rivero González et al. (2012) appears to be strongly $\mathrm{N}$-enriched, and a clear correlation of nitrogen and helium enrichment was found. 
The so far - in terms of proper statistical treatment - most comprehensive search for correlations among many variables in OB stars has been performed by Aerts et al. (2014), finding that $v \sin i$ and the rotational frequency, as well as the magnetic field strength have no predictive power for nitrogen abundances. Furthermore, comprehensive comparisons of observational constraints extracted from numerous literature sources with predictions from various stellar evolution models were presented by Martins \& Palacios (2013). Note that both studies draw their conclusions from highly heterogeneous observational datasets; these may require (partial) revision in view of the following discussion.

\section{Observational challenges for OB stars}

Quantitative analyses of OB stars are not only demanding in terms of model atmospheres and line formation, they also have to face several observational challenges.

Multi-object spectroscopy has improved the efficiency of cluster studies enormously, however it brings its own challenges, as experienced e.g. within the FLAMES Survey of Massive Stars (Evans et al. 2005). Crucial issues are the large dynamical range between faint main-sequence stars and bright (super)giants, which can span $\sim 4$ to 7 mag in $V$ (this can be solved by observing a few objects with a different spectrograph), and fibre/slit positioning limitations within the field-of-view. The latter is in particular a problem for observations of distant clusters like in the Magellanic Clouds, where the sampling of objects in the denser parts of a cluster may in fact be sparse (Evans et al. 2006). Instead, many objects from the field surrounding the cluster may be covered.

The probably most important challenge for observations of OB stars is the identification of contamination of a spectrum by light from another star. It has turned out in the past years that the binary fraction among OB stars may reach 50-70\% (Sana et al. 2012; Chini et al. 2012), many of which show SB2 characteristics. Signatures for the presence of second light may be subtle and can often be recognised only in high-resolution, high-S/N spectra. Second light changes line depths relative to the continuum flux and thus also equivalent widths. An analysis of a binary composite spectrum with standard techniques - therefore assuming it to be single - results in erroneous stellar parameters and elemental abundances. However, a correct analysis can be achieved using either spectrum disentangling (e.g. Pavlovski \& Southworth 2013) or spectrum synthesis accounting for both sources (e.g. Irrgang et al. 2014).

Chemically peculiar stars are rare among OB stars and comprise He-rich stars and Heweak stars (see e.g. Smith 1996 for a discussion). They are of limited use for obtaining observational constraints on massive star evolution, as their atmospheres show either spotted elemental concentrations, or vertical stratification because of atomic diffusion resulting from the interplay of radiative levitation and gravitational settling.

Finally, second light from the disk complicates the analysis of Be stars. When continuum and line emission from a disc overlaps with the stellar spectrum, this gives rise to a variety of spectral morphologies depending on disc parameters, disc size, and viewing angle on the star+disc system (see e.g. Rivinius et al. 2013). Moreover, Be-stars rotate close to the breakup velocity, leading to a distortion from spherical geometry and non-uniform surface temperatures (hot poles and cool equator) and densities. At present, analyses based on a comprehensive model of the stellar atmosphere+disk cannot be performed, therefore any observational constraints suffer from limited accuracy and precision.

Any star sample to be analysed with standard model atmosphere techniques needs to be carefully checked for the presence of binary, chemically peculiar or Be stars. Such objects need to be excluded in order to avoid the introduction of observational bias. 


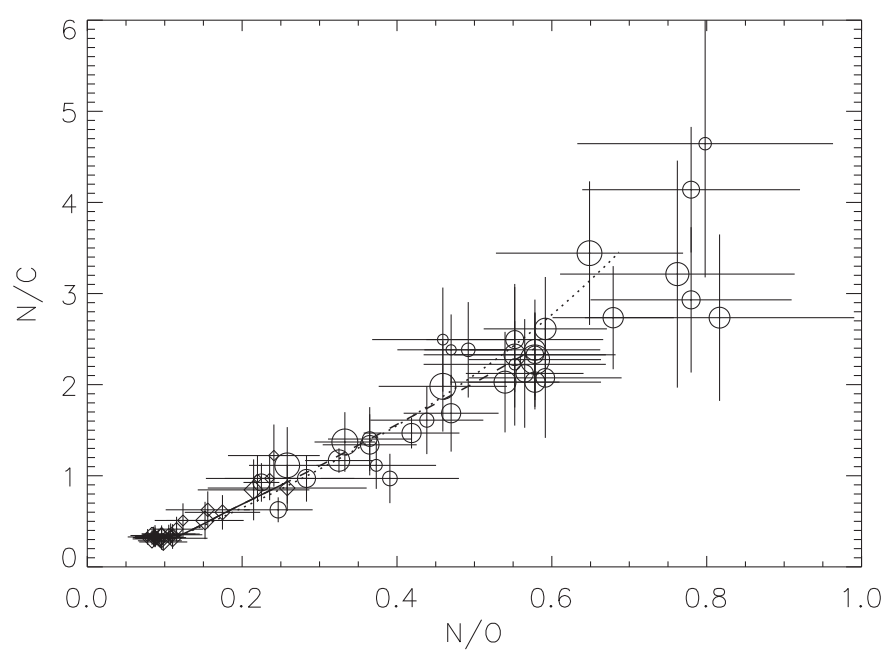

Figure 1. N/C vs. N/O abundance ratios (by mass) for homogeneously analysed stars from the samples of Przybilla et al. (2010), Nieva \& Przybilla (2012) and Nieva \& Simón-Díaz (2011), and Firnstein \& Przybilla (2012, including preliminary data). B-type main-sequence stars are displayed as diamonds, BA-type supergiants as circles. The symbol size encodes the stellar mass and error bars give $1 \sigma$-uncertainties. The lines represent predictions from evolution calculations, for a rotating $15 M_{\odot}$ star, $v_{\text {rot }}^{\text {ini }}=300 \mathrm{~km} \mathrm{~s}^{-1}$, (Meynet \& Maeder 2003, solid line: until the end of the main sequence; dashed line: until the end of He burning) and for a star of the same mass and $v_{\mathrm{rot}}^{\text {ini }}$ that in addition takes the interaction of rotation and a magnetic dynamo into account (Maeder \& Meynet 2005, dotted line: until the end of the main sequence). Note the clump of 20 stars at the base of the tracks that results from objects with pristine CNO abundances.

\section{New developments in the quantitative spectroscopy of OB stars}

The question of the presence of systematical bias in observational constraints is a crucial one for the comparison with stellar evolution models. Such bias can be of observational nature (see above) or it can be produced by flaws in the spectral analysis, and - at first glance - it may appear difficult to establish the presence of bias. However, a simple and objective test exists, which can be used to assess the accuracy and precision of analysis results: the N/C vs. N/O diagram (Przybilla et al. 2010; Maeder et al. 2014).

The ratios of the participants in the CNO cycles at the stellar surface depend both on the changes produced by nuclear reactions and the dilution effects produced by mixing. Analytical relations can be derived for the predicted nuclear path, which indicate an initially almost linear slope of e.g. $\mathrm{d}(\mathrm{N} / \mathrm{C}) / \mathrm{d}(\mathrm{N} / \mathrm{O}) \approx 4$ for typical pristine $\mathrm{CNO}$ abundances as found in the solar neighbourhood at present day (Przybilla et al. 2008; Nieva \& Przybilla 2012), which is matched well by detailed stellar evolution models. In particular, the $\mathrm{N} / \mathrm{C}$ vs. N/O plot shows little dependence on the initial stellar masses, rotation velocities, and nature of the mixing processes up to relative enrichment of N/O by a factor of about four. Figure 1 shows an example how tight is the match between a homogeneously analysed star sample with high-quality spectra - that have been scrutinised in terms of observational bias - and the predictions by stellar evolution models. The observational sample consists of 29 early B-stars on the main sequence and 35 BA-type supergiants of similar stellar masses, which have been analysed using well-tested hybrid non-LTE models (Nieva \& Przybilla 2007; Przybilla et al. 2006, 2011), an iterative analysis technique (Nieva \& Przybilla 2008) that employs almost the entire observed spectrum and a consistent set of comprehensive model atoms (for CNO: Przybilla et al. 2000, 2001; Przybilla \& Butler 2001; Nieva \& Przybilla 2008; Becker \& Butler 1988, the latter updated). 


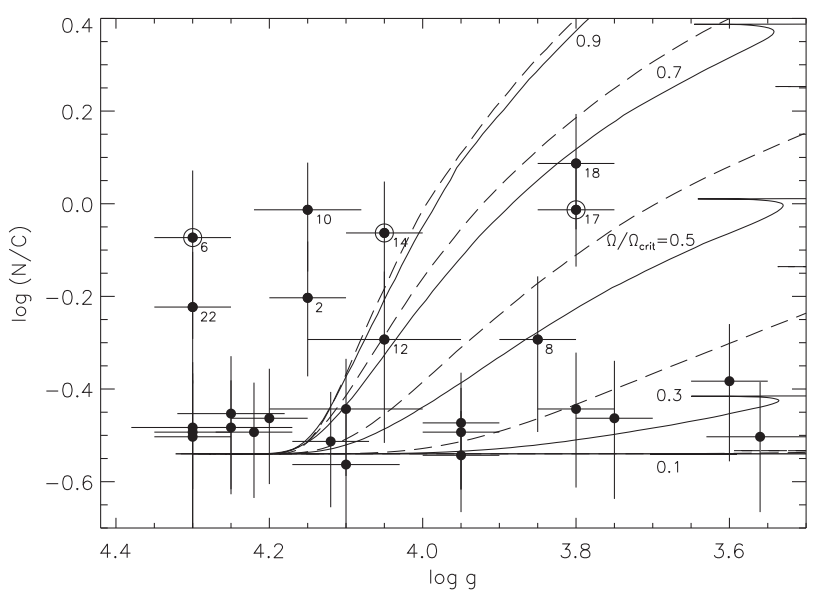

Figure 2. Sample stars of Nieva \& Przybilla (2014, dots) in the $\log g-\log (N / C)$-plane. Predictions from evolution models for rotating stars by Georgy et al. (2013) are shown for a $9 M_{\odot}$ (full) and a $15 M_{\odot}$ model (dashed line) at $Z=0.014$, respectively, for initial $\Omega / \Omega_{\text {crit }}$ varying from 0.1 to 0.9 , as indicated. About $1 / 3$ of the observed objects show signatures of CN-mixing $(\log (\mathrm{N} / \mathrm{C}) \gtrsim-0.3)$. Objects \#6, 14 and 17 are magnetic.

Both, high accuracy and precision can be achieved in this approach, with $1 \sigma$-uncertainties reduced to $1-2 \%$ in $T_{\text {eff }}$, to $0.05-0.10$ dex in surface gravity $\log g$ and $\sim 10-20 \%$ for absolute elemental abundances. Thus, almost the entire observed visual and near-IR spectra can be reliably reproduced and various additional constraints are also met, like a good match of the observed spectral energy distribution or the good agreement between spectroscopic and Hipparcos parallaxes (Nieva \& Przybilla 2012). The most important finding from this study is that young massive stars in the solar neighbourhood show a high degree of chemical homogeneity (except in nitrogen abundance), facilitating a presentday cosmic abundance standard (CAS) to be established. Moreover, evolutionary masses, radii and luminosities can be determined in this approach to better than typically $5 \%$, $10 \%$, and $20 \%$ uncertainty, respectively, allowing the mass-radius and mass-luminosity relationships to be recovered for single core H-burning objects with a similar precision (within a factor 2-4) as derived from detached eclipsing binaries (Nieva \& Przybilla 2014).

While the N/C vs. N/O diagram provides a simple quality test for observational results, it is not suited itself for assessing the quality of stellar evolution models. This has to be done by other means, e.g. employing the $\log g-\log (\mathrm{N} / \mathrm{C})$ diagram (see Fig. 2), which represents a proxy for the evolution of the surface abundance as a function of the evolutionary age of a star. The models evolve in Fig. 2 from the zero-age main sequence on the left to the terminal-age main sequence (the hook at the right), with stars of higher initial mass and/or higher angular velocity $\Omega$ producing stronger mixing. Noticeable effects of mixing are predicted by the particular models to occur when the stars attain $\log g$-values in the range $\sim 4.0$ to 3.9 for ratios of initial to critical angular velocity $\Omega / \Omega_{\text {crit }} \gtrsim 0.4$. In this case, most of the apparently slowly-rotating sample stars are consistent with the model predictions: many appear unmixed and some more evolved objects show signatures of mixing within the expectations. However, for a small group of apparently unevolved stars with low $v \sin i(\# 2,6,10,22)$ other scenarios have to be invoked to explain their degree of mixing, possibly related to binarity and/or magnetic fields. In particular, the unusual characteristics of $\tau$ Sco $(\# 6)$ point to a blue straggler nature, due to a binary merger (Nieva \& Przybilla 2014). Obviously, further such high-quality investigations are required, encompassing also faster-rotating stars to obtain reliable conclusions. 


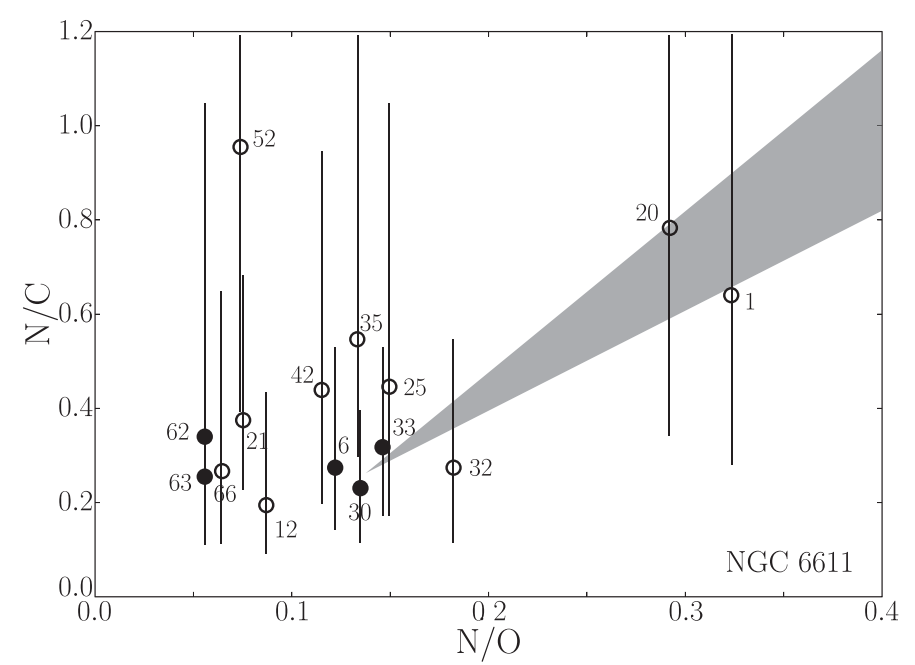

Figure 3. N/C vs. N/O abundance ratios (by number) in the Galactic open cluster NGC 6611 from observations by Hunter et al. (2009). The shaded area shows the domain predicted by the stellar models of Ekström et al. (2012), between 5 and $60 M_{\odot}$ at $Z=0.014$. Error bars on the $N / O$ ratios are of the same order of magnitude as those shown for the $N / C$ ratios. The numbers attached to the data points correspond to the identifiers employed within the FLAMES Survey. Normal stars are marked by a filled symbol, binaries (or binaries candidates) or Be stars, i.e. stars with biased results, by an open symbol. According to Maeder et al. (2014).

At this point we can resume the discussion of the reliability of observational constraints reported in the literature so far (see Sect. 3). Unfortunately, abundances of all three elements carbon, nitrogen and oxygen are rarely provided. However, for the important FLAMES Massive Star Survey, one can perform the test employing the N/C vs. N/O diagram. Overall, there is agreement of the data with model predictions within the (large) observational uncertainties (Maeder et al. 2014), see also Fig. 3, but the underlying scatter is much larger than one may expect from considering changes in the initial mass, rotation, and model details on the nuclear path. In order to assess the significance of the scatter, one can aim at confirming the results by Hunter et al. (2009). This can be achieved under the premise that no matter the details of the model implementations, similar analysis results will be derived if they are sufficiently realistic. In particular, a good match of the synthetic with the observed spectra should be obtained globally, and in detail, as demonstrated by Nieva \& Przybilla (2012). Thus, model spectra computed with the methods introduced earlier in this Section for atmospheric parameters and abundances as derived by Hunter et al. (2009) are expected to match the observed spectra from the FLAMES Survey, which are publically available. The test was made for a sub-sample of stars, including all stars in the Galactic cluster NGC 6611 analysed by the FLAMES Survey. Again, an overall good match was found for many spectral features. However, a close inspection of the observed spectra showed the presence of observational bias: many stars were identified as (candidate) SB2 systems, some objects turned out as Be stars and one chemically peculiar He-strong star was identified. For the case of NGC 6611 all the potentially biased stars are identified in Fig. 3, which consequently should be removed from the interpretation, reducing the observed scatter. The main conclusion from the re-assessment by Maeder et al. (2014) is that a careful and critical re-investigation of the FLAMES Survey data presented by Hunter et al. (2009) is clearly required. This may resolve the unusual characteristics of chemical abundances of the FLAMES Survey objects when compared to other studies of Galactic stars (Martins \& Palacios 2013). 
In the case of the heterogeneous sample of observational constraints collected by Aerts et al. (2014), full CNO abundances are not available. However, there are other indicators for the presence of bias. The nitrogen abundances scatter by over 2 dex; in particular, the presence of a considerable fraction of N-poor OB stars in the solar neighbourhood, some objects with values of $\sim 1 / 10$ of the CAS baseline abundance (i.e. comparable to the metallicity of halo objects), suggest that some of the data needs re-evaluation.

\section{Conclusions \& Recommendations}

In summary, it is found that the currently available observational constraints for testing stellar evolution models suffer from the presence of bias in many cases. The data clearly support that mixing of the surface layers with CNO-processed material has taken place in some stars, however their precision and accuracy is often insufficient to distinguish between different stellar evolution models. There exists a class of slowly-rotating massive stars on the main sequence that shows evidence for mixing, unexplained by standard evolution of single rotating stars. The available data also implies that binary interactions, including mergers, need to be considered for explaining the properties of a sub-set of stars.

Currently, the exploration of the effects of magnetic fields on the transport of angular momentum and chemical elements in massive stars is just beginning. Efforts need to be undertaken to characterise the properties of the $\sim 70$ magnetic OB-stars known so far as accurate and precise as possible in a homogeneous way. Moreover, existing surveys need to be continued to search for additional objects in order to improve the still poor number statistics. This is because of the different potential formation channels and many variables involved. Population synthesis (and therefore a large database) is required if one aims at establishing correlations between surface magnetic fields, abundances and surface rotation. It will also be necessary to extend the analysed star samples to both apparently single stars and objects identified as binaries, irrespective of $v \sin i$. The complexity and scope of the topic is such that the collaboration of both large teams currently working on this (BOB, MiMeS) will be required to achieve breakthrough results.

\section{Acknowledgements}

I would like to thank M.-F. Nieva, A. Irrgang, M. Firnstein and K. Butler for collaboration and discussions on the quantitative spectroscopy of massive stars over the past years, and A. Maeder, G. Meynet, S. Ekström, P. Eggenberger and C. Georgy for stimulating insights into the interpretation of these findings in the context of stellar evolution.

\section{References}

Aerts, C., Molenberghs, G., Kenward, M. G., \& Neiner, C. 2014, ApJ 781, 88

Becker, S. R. \& Butler, K. 1988, A\&SA 201, 232

Bouret, J.-C., Donati, J.-F., Martins, F., et al. 2008, MNRAS 389, 75

Brott, I., de Mink, S. E., Cantiello, M., et al. 2011, A\&A 530, A115

Cantiello, M., Langer, N., Brott, I., et al. 2009, A\& $A$ 499, 279

Chieffi, A. \& Limongi, M. 2013, ApJ 764, 21

Chini, R., Hoffmeister, V. H., Nasseri, A., Stahl, O., \& Zinnecker, H. 2012, MNRAS 424, 1925

Donati, J.-F., Howarth, I. D., Jardine, M. M., et al. 2006, MNRAS 370, 629

Ekström, S., Georgy, C., Eggenberger, P., et al. 2012, A\&A 537, A146

Evans, C. J., Lennon, D. J., Smartt, S. J., \& Trundle, C. 2006, A\&A 456, 623

Evans, C. J., Smartt, S. J., Lee, J.-K., et al. 2005, A\&A 437, 467

Firnstein, M. \& Przybilla, N. 2012, A\&A 543, A80

Fossati, L., Zwintz, K., Castro, N., et al. 2014, A\&A 562, A143 
Frischknecht, U., Hirschi, R., Meynet, G., et al. 2010, A 6 A 522, A39

Georgy, C., Ekström, S., Granada, A., et al. 2013, A\&A 553, A24

Georgy, C., Granada, A., Ekström, S., et al. 2014, A\&A 566, A21

Heger, A. \& Langer, N. 2000, ApJ 544, 1016

Heger, A., Woosley, S. E., \& Spruit, H. C. 2005, ApJ 626, 350

Henrichs, H. F., de Jong, J. A., Verdugo, E., et al. 2013, A\&A 555, A46

Henrichs, H. F., Kolenberg, K., Plaggenborg, B., et al. 2012, A $6 A$ 545, A119

Hubrig, S., Schöller, M., Kharchenko, N. V., et al. 2011, A\& A 528, A151

Hummel, C. A., Rivinius, T., Nieva, M.-F., et al. 2013, A\&A A 554, A52

Hunter, I., Brott, I., Langer, N., et al. 2009, A $\mathscr{G} A$ 496, 841

Irrgang, A., Przybilla, N., Heber, U., et al. 2014, A\& $A$ 565, A63

Langer, N. 2014, in IAU Symposium Ser., Vol. 302, p. 1

Maeder, A. \& Meynet, G. 2005, A\&A 440, 1041

Maeder, A., Meynet, G., Ekström, S., \& Georgy, C. 2009, Comm. Asteroseism. 158, 72

Maeder, A., Przybilla, N., Nieva, M.-F., et al. 2014, A\&A 565, A39

Martins, F., Escolano, C., Wade, G. A., et al. 2012a, A\&GA 538, A29

Martins, F., Mahy, L., Hillier, D. J., \& Rauw, G. 2012b, A\&A 538, A39

Martins, F. \& Palacios, A. 2013, A\&A 560, A16

Meynet, G., Eggenberger, P., \& Maeder, A. 2011, A\& $A$ 525, L11

Meynet, G. \& Maeder, A. 2000, A\& $A$ 361, 101

Meynet, G. \& Maeder, A. 2003, A\& A 404, 975

Morel, T. 2011, Bulletin de la Societe Royale des Sciences de Liege 80, 405

Morel, T. 2012, ASP Conf. Ser. 465, p. 54

Morel, T., Hubrig, S., \& Briquet, M. 2008, A\& A 481, 453

Neiner, C., Alecian, E., Briquet, M., et al. 2012, A\&A 537, A148

Nieva, M. F. \& Przybilla, N. 2007, A\&A 467, 295

Nieva, M. F. \& Przybilla, N. 2008, A\&SA 481, 199

Nieva, M.-F. \& Przybilla, N. 2012, A\& A 539, A143

Nieva, M.-F. \& Przybilla, N. 2014, A\&A 566, A7

Nieva, M.-F. \& Simón-Díaz, S. 2011, A\&A 532, A2

Pavlovski, K. \& Southworth, J. 2013, in EAS Publ. Ser. 64, p. 29

Petit, V., Owocki, S. P., Wade, G. A., et al. 2013, MNRAS 429, 398

Przybilla, N. \& Butler, K. 2001, A\&A 379, 955

Przybilla, N., Butler, K., Becker, S. R., \& Kudritzki, R. P. 2006, A\&A 445, 1099

Przybilla, N., Butler, K., Becker, S. R., Kudritzki, R. P., \& Venn, K. A. 2000, A\&3A 359, 1085

Przybilla, N., Butler, K., \& Kudritzki, R. P. 2001, A\&GA 379, 936

Przybilla, N., Firnstein, M., Nieva, M. F., Meynet, G., \& Maeder, A. 2010, A\&A 517, A38

Przybilla, N., Nieva, M.-F., \& Butler, K. 2008, ApJ (Letters) 688, L103

Przybilla, N., Nieva, M.-F., \& Butler, K. 2011, J. Phys. Conf. Ser. 328, 012015

Rivero González, J. G., Puls, J., Najarro, F., \& Brott, I. 2012, A\&SA 537, A79

Rivinius, T., Carciofi, A. C., \& Martayan, C. 2013, A\&A Rev. 21, 69

Sana, H., de Mink, S. E., de Koter, A., et al. 2012, Science 337, 444

Schneider, F. R. N., Langer, N., de Koter, A., et al. 2014, A\& A, 570, A66

Smith, K. C. 1996, ApESSS 237, 77

Spruit, H. C. 2002, A\&A 381, 923

Ud-Doula, A., Owocki, S. P., \& Townsend, R. H. D. 2009, MNRAS 392, 1022

Wade, G. A., Grunhut, J., Alecian, E., et al. 2014, in IAU Symposium Ser., Vol. 302, p. 265

Wade, G. A., Maíz Apellániz, J., Martins, F., et al. 2012, MNRAS 425, 1278

\section{Discussion}

ANDERSON: Do you think using the $\frac{N / C}{N / O}$ ratio could be a viable constraint to improve the accuracy of statistical analyses of CNO abundances such as that by the FLAMES Survey? 
PRZYBILla: The $\frac{N / C}{N / O}$ ratios for a star sample should follow tightly the predicted nuclear path. This is an objective quality indicator for stellar analyses, and it can be used to detect potential systematic error in the analyses. Efforts should therefore be made not only to derive nitrogen abundances - undoubtedly the most sensitive indicator for chemical mixing - but also carbon and oxygen abundances.

AERTS: Completely agreed that we would like to re-apply the methodology of Aerts et al. (2014, ApJ, 781, 88) on a precise and accurate sample, but we did the best we could to assemble a large enough database at the present day...

PULS: A comment: In order to apply the correlation analysis (Aerts et al.) successfully, we cannot only use B-type objects which can be analysed with a very high precision, but also the more massive/hotter stars. For these objects the errors become necessarily larger (mass-loss, complete NLTE description, etc.).

SUNDQUIST: I admit I do not fully understand the high accuracy (as opposed to precision) you seem to claim in your abundances. To me, systematic uncertainties in atomic data and model atmospheres should enter here. Could you perhaps comment on this?

PRZYBILla: We have made extensive comparisons of different model atmospheres and found overall good agreement in the stratifications and emerging fluxes for the kind of stars under study. We also investigated the effects of varying input atomic data for the analyses. The resulting systematic uncertainties from uncertainties in the atmospheric parameters and atomic data typically amount to $\sim 0.07-0.12$ dex in abundance (depending on element and stellar parameters), which is comparable to the $1 \sigma$ statistical uncertainties $(\sim 0.05-0.10$ dex $)$.

NAJARRO: 1) Could you comment on systematics for the $\log g, T_{\text {eff }}$ determinations for the B0 and O9 V's? 2) Are your high log g, highly N-rich data early-type (one was $\tau$ Sco)?

PrZYBILla: 1) Admittedly, the analysis becomes more complicated for the B0 Vs and in particular for (weak-wind) O9 Vs. Overall, the scatter in the abundances increases, but the ability to establish still multiple ionization equilibria simultaneously raises hope that the stellar parameter determination is not affected seriously by systematic error. We start to see systematic deviations in abundances from some lines of some elements, indicating that the employed models are just about sufficiently realistic. And, we are still missing several of the higher ionization stages that would be useful for the analysis. Work on this is in progress. 2) The fraction of high $\log g$, N-rich objects among the stars with spectral type earlier than B0.5 is about the same as elsewhere in the sample, $\sim 1 / 3$.

MAssEY: Just one comment. These fits you showed are beautiful. It forces me to remember the question raised by Georges in the general discussion Tuesday: are the analysis pipelines used in large survey sufficiently accurate? I know of recent large studies of finer data that have used automatic fitting techniques to find the "best" fits - but these best fits are not necessarily good fits. The quality of the fit must also be considered.

Herrero: I wanted to follow Phil's comment before and say that we need pipelines for data analysis if we want to have large amounts of data and good statistics. We then have to be open and provide all the information, check carefully all aspects and use the pipelines not as black boxes. Then we will learn from our mistakes and improve our badly needed pipelines. 